\title{
Numerical Study of the Flow past a Rotating Cylinder at Supercritical Reynolds Number
}

\author{
Q. Yao, C.Y. Zhou \& C. Wang* \\ Harbin Institute of Technology Shenzhen Graduate School, Shenzhen, China \\ *Corresponding author: C. Wang
}

ABSTRACT: The rapid development of the large-scale equipment promotes the investigation on flow around rotating cylindrical aerodynamic characteristics at supercritical Reynolds number. In this paper, the flow past a rotating cylinder at supercritical Reynolds number has been studied using the computational fluid dynamic methods where the $\mathrm{k}^{-} \varepsilon$ turbulence model is adopted. It is found that the flow around the rotating cylinder changing law of flow field have been investigated, lift and drag force characteristics have also been analysis at supercritical Reynolds number condition.

KEYWORD: Rotating cylinder; Supercritical Reynolds number; Velocity ratio; Lift and drag force

\section{INTRODUCTION}

As a typical problem for understanding flow around bluff bodies, flow past a rotating circular cylinder has been investigated by many researchers over centuries, based on theoretical, experimental and numerical methods. The cylinder which rotates itself and translates in a flow field will generate a great lift force. Researchers have been committed to use this large lift force in practice, such as the wings, the space shuttle, the wind, the ship and so on. However, because there is no theoretical support, most applications are all in testing stage. With the increasing size of these engineering structures, the aerodynamic characteristics of the flow past a rotating cylinder at high Reynolds number or even ultrahigh Reynolds number need to be studied. But research in this field has not been recorded, mostly are just some research at low Reynolds number.

Magnus (1853) confirmed that the combined effects of ration and translation lead to the nonequivalence of the both sides of the cylinder in the flow direction through experimental research. He put forward that the migration directions of a rotating object is different based on its rotating direction. This phenomenon is called the Magnus effect. Prandtl (1926) conducted visual experiment on the flow past a cylinder for the first time. He proposed that the maximum lift force coefficient $C_{l}$ which generated by a rotating cylinder was $4 \pi$. Glauert (1957) considered that the shearing in boundary layer increased with the increasing of the cylinder's rotating speed. When the vorticity on the cylinder's surface accumulated large enough, it would pass through the closed flow line under the effect of centrifugal force and then exfoliated towards the wake. So the lift and drag force coefficient would exceed $4 \pi$, and increased with the speed increasing. He also explained the main reason that the lift force coefficient measured in experiment could not exceed $4 \pi$ was the influence of three-dimensional effect. Swanson (1961) provided an excellent overview on Magnus effect and a summary of investigation. The author also designed an experimental device which could reduce the three-dimensional effect, and he conducted lots of experiments. Diaz et al. (1985) studied two-dimensional flow past a rotating cylinder when $R e=9000$ through experiments. The conclusion was when the circumferential velocity was less than the speed of the flow field, the Karman vortex street existed clear in the wake; when the circumferential velocity was more than the speed of the flow field but less than its twice, the Karman vortex street was restrained; and when the circumferential velocity was more than twice of the speed of the flow field, the Karman vortex street disappeared. Coutanceau (1985) studied the vertex's formation, development and shedding processes in a rotating cylinder's wake when $R e<1000$ and velocity ratio $\alpha$ was $0 \sim 3.25$. Chew (1995) used numerical method to study the two-dimensional flow past a cylinder under unsteady condition when $R e=1000$. The velocity ratio ranged from 0 to 6 . The results showed that the critical velocity ratio suppressed the generation of Karman Vortex Street was 2. Nair (1998) studied the flow past a cylinder under high Reynolds number, and he thought the limit of lift force coefficient could exceed $4 \pi$. Kang (1999) calculated the flow past a rotating cylinder when $\mathrm{Re}=60,100,160$ and the velocity ratio $\alpha$ ranged from 0 to 6 . The results showed that $C_{l}$ increased with the increase of $\alpha$. On the contrary, $C_{d}$ reduced with the increase of $\alpha$. Mittal (2004) conducted lot of numerical study, and he considered the main factors which affected the lift and drag force of a rotating cylinder were its end face condition and aspect ratio. Karabelas (2010) 
conducted LES numerical simulation under high Re, and analyzed the morphological changes of the flow field at different $\alpha$. There were linearly increasing relationship between $C_{l}$ and $\alpha . C_{d}$ reduced with the reduction of $\alpha$.

The open literature is poor in rotating cylinder flows at high Re regimes, and this article conduct numerical simulation on two-dimensional flow past a rotating cylinder at ultrahigh Reynolds number. The main works are: (1) obtain the flow field's cloud images under different velocity ratio at ultrahigh Reynolds number, analyze the influence law of the velocity ratio to the flow field, and try to analyze the change of a cylinder's lift force caused by different flow field's change. (2) obtain the changing relation between lift force coefficient and velocity ratio at supercritical Reynolds number; (3) obtain the changing relations between drag force coefficient and each parameter at supercritical Reynolds number.

\section{COMPUTATIONAL SETUP}

This paper uses mixed grids which are shown in Figure 1. It uses non-structural grid close to the wall to improve the calculation precision, and uses structural grid far away from the wall to reduce computing time. Size of the cell gradually increases as the distance from surface of the cylinder becomes longer.

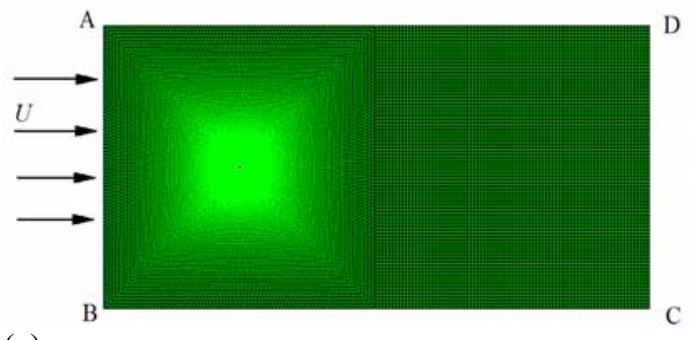

(a)
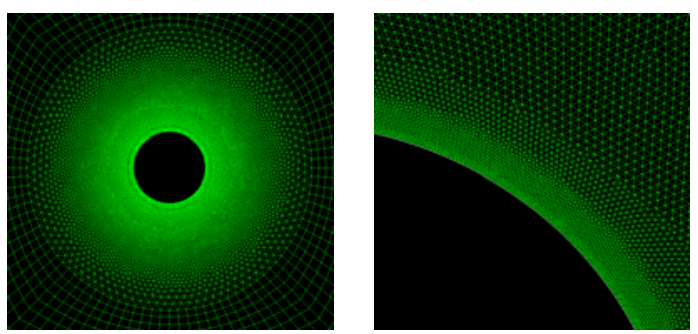

(b)

(c)

Figure 1. Computational domain and grids. (a) Computational domain is $80 \mathrm{D} \times 160 \mathrm{D}$, number of grids are almost 120000 , AB is set to velocity inlet, $\mathrm{AD}, \mathrm{BC}$ are set to symmetry, $\mathrm{CD}$ is set to pressure outlet, (b) hybrid with structural and unstructured grids, unstructured grids are used closed to the surface of cylinder, the others are used structural, cylinder rotates in the clockwise direction, (c) detail of grids closed to surface.

Numerical simulations in this paper use fluid dynamic software FLUENT 6.3. Turbulence model

chooses RNG $k-\varepsilon$ model with standard wall treatment, and this model simulates rotational motion very good. The boundary conditions are set as follows: $\mathrm{AB}$ is velocity in-let, and $\mathrm{AD}, \mathrm{BC}$ are symmetry, and $C D$ is pressure outlet. The flow direction is shown in the Figure 1, and the cylinder rotates clockwise.

\section{RESULTS AND DISCUSSION}

\subsection{Basic flow patterns}

Fig. 2 shows the diagram of velocity flow field of stationary and rotating cylinder at $\mathrm{Re}=1.3 \times 10^{6}$. When the cylinder is stationary, the streamline of velocity as shown in Fig.2 (a), stagnation located at 180 degree, and Karman Vortex Street generated. When the cylinder clockwise rotation speed was set as 3000rpm, the streamline of velocity shown in Fig. 2(b), the Karman Vortex Street disappeared in the rear, instead by standing vortex.

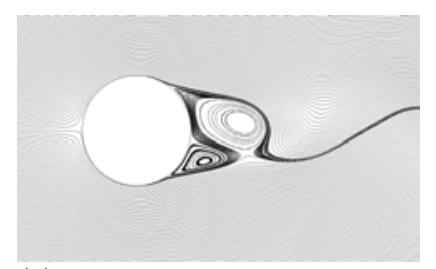

(a)

Figure 2. Diagram of flow field velocity a) Streamline of flow past a stationary cylinder; b) Streamline of flow past a rotating cylinder.

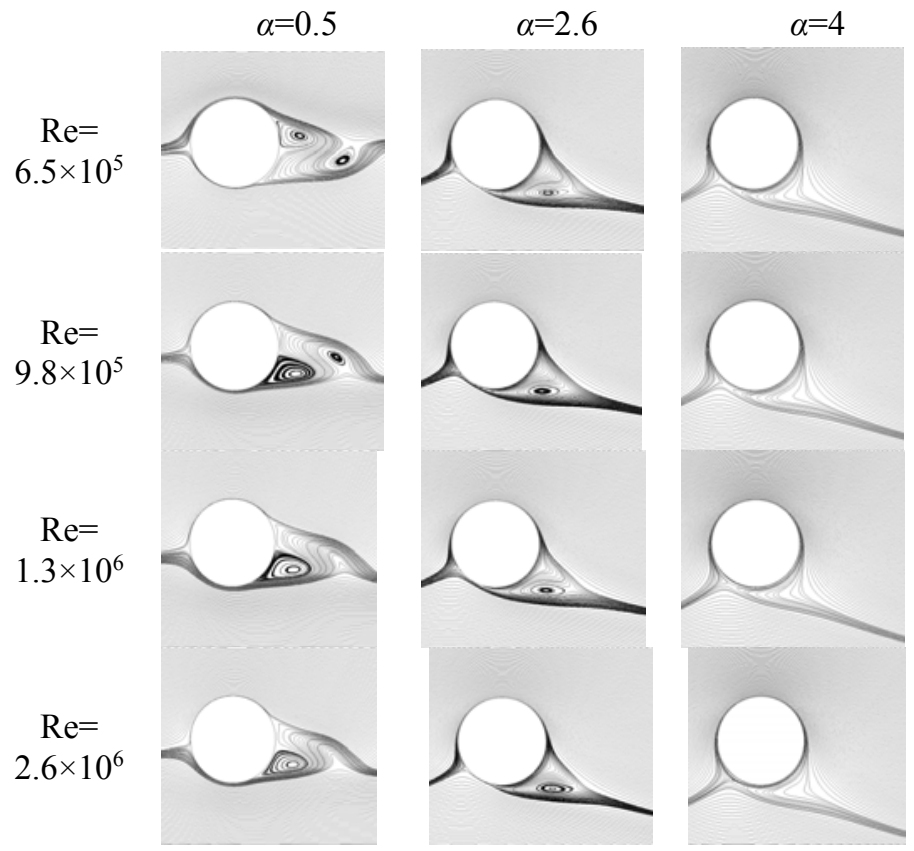

Figure. 3 Variations flow field of rotating cylinder influenced by Re numbers and peripheral speed ratio $\alpha$.

Figure 3 shows the flow field of rotating cylinder influenced by Re numbers and peripheral speed ratio 
$\alpha$. The cylinder rotated clockwise and flow from left to right. Symmetry was destroyed by rotating, and boundary layer separated ahead, caused Karman Vortex Street beginning to disappear. According to the knowledge of fluid mechanics, the flow field is divided into a cylindrical pressure resistance and friction resistance. With the peripheral speed ratio increased, pressure at front and rear sides of the cylinder became symmetrical, and the resistance coefficient reduced. So, the influence parameters of resistance coefficient on cylinder were the stagnation and the tail vortex.

\subsection{Variation of Lift and Drag Force Coefficients}

Re and $\alpha$ are two important parameters in the flow past a rotating cylinder. In order to study the change of the flow field with these two parameters, we do some research in this section which is shown in Figure 4.

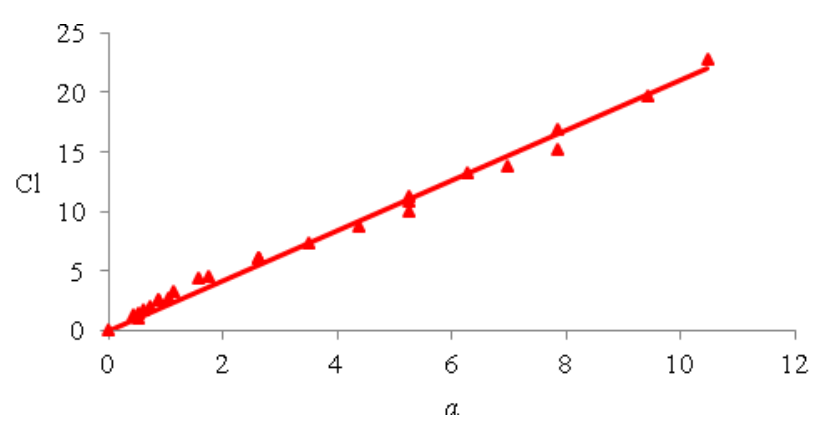

Figure 4. Coefficient of lift force changing curve influenced by peripheral speed ratio $\alpha$.

From the formula $C_{l}=2 \pi \Omega R / U$ which is derived from the Kutta-Joukowski theorem that the parameter $C_{1}$ has a relationship with parameters $\Omega, R$ and $U$. Their relationships are shown in Figure 4 . We can find there does present a linear relationship between $C_{l}$ and $\alpha$, and the former is almost twice of the latter.

The relationships between $C_{d}$ and $\operatorname{Re}$ and $\alpha$ are complex. Scholars have only found the relation between $\mathrm{C}_{\mathrm{d}}$ and $\mathrm{Re}$ in the condition of stationary cylinders with small Re number through formula derivation. To analyze the relation between $C_{d}$ and $\alpha$, scholars deduced different conclusions with the methods of numerical analysis and experiment, etc. After a simulation of the condition with same speed and different Re numbers, an interesting conclusion is established. As shown in Figure. 5, when the speed are same, the changing trend between drag coefficient with $\mathrm{Re}$ is same as that of Re in the condition of static cylinders. From the formulas $R e=\rho U d / \mu$ and $\alpha=\omega r / U$ we can easily find that the effects of streams wind speed $U$ on $R e$ and $\alpha$ are adverse and the parameter $U$ is the most significant impact factor of $C_{d}$.
The influences of wind speed, diameter and rotational speed on $C_{d}$ are shown in Figure 6 and Figure 7. We can see from the figures that the changing trend between drag coefficient with wind speed is similar as that of diameter. Combined with the formula $R e=\rho v d / \mu$, we can get the relationship between drag coefficient and Re number, as shown in Figure 5. The changing trend between drag coefficient and Re number under different wind speeds is in accordance with that under different diameters, both getting the smallest drag coefficient when $\mathrm{Re}$ number is $2 \times 10^{6}$. In the former condition the smallest drag coefficient is 0.151 and in the latter condition it's 0.164 . The changing trend is similar as shown in Fig.5. What's different is that the smallest drag coefficient is 0.35 when the cylinder is static while is 0.16 when the cylinder is rotating. Another difference is that Re number changes from $5 \times 10^{5}$ to $2 \times 10^{6}$ when the smallest drag coefficient is got.

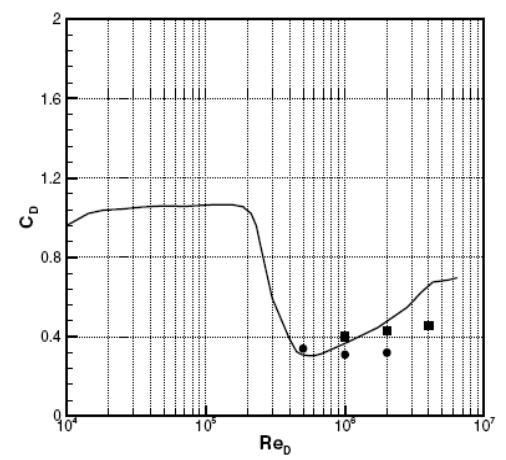

a) $\mathrm{Cd}$ changing curves of stationary cylinder (Achenbach, 1968)

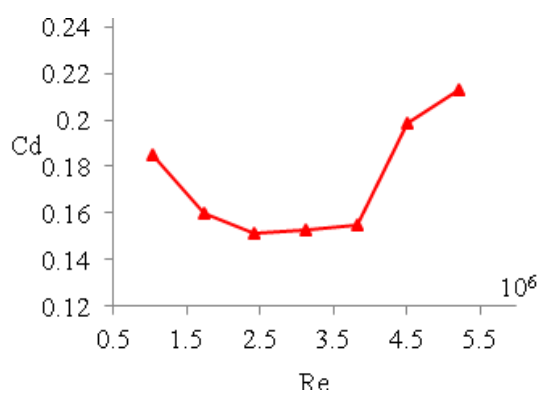

b) Cd changing curves of rotating cylinder

Figure 5. Coefficient of drag force changing curves influenced by Re.

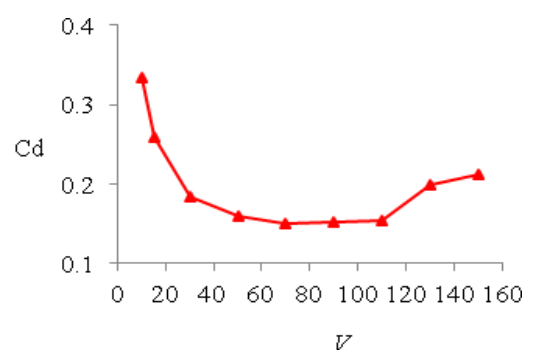

Figure 6. Coefficient of drag force changing curves influenced by wind speeds $\mathrm{V}$. 


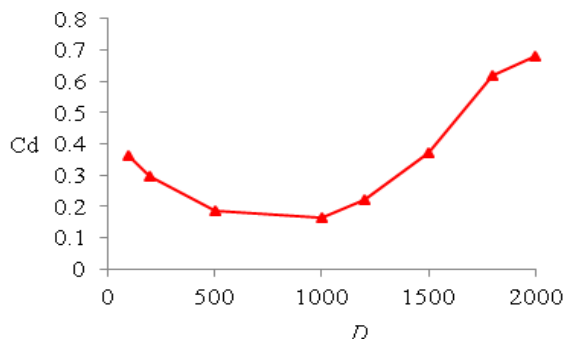

Figure 7. Coefficient of drag force changing curves influenced by diameters $\mathrm{D}$.

Figure 8 shows the relationship between $C_{d}$ and rotating speed. Combined with Fig. 2 we can find that pre-stagnation and post-stagnation points slip down and make pressure difference before and after the cylinder decrease until the symmetric flow field is formed, the pressure drag, which is mainly frictional resistance, is smallest. The frictional resistance caused by boundary layer of the rotating cylinder is low and doesn't change too much with the increase of the rotating speed.

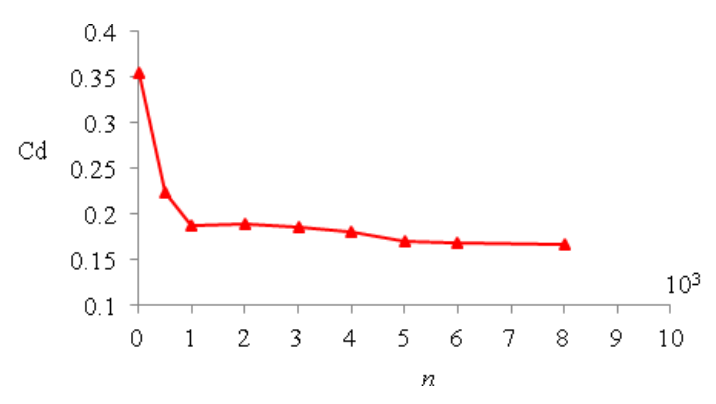

Figure. 8 Coefficient of drag force changing curves influenced by rotating speed $n$.

From the simulation result we can hardly secure the relationship between $C_{d}$ and $\alpha$ and that's why different change laws are deduced by scholars. We should analyze the changing law of $C_{d}$ form the relation between $\mathrm{Re}$ and rotating speed.

\section{CONCLUSION}

In this paper, the numerical method to simulate the flow around rotating cylinder at supercritical Reynolds number. Firstly, the variation of flow field around a rotating cylinder have been analysis, Karman vortex street will re-appear along with the Reynolds number increaseing, as the peripheral speed ratio $\alpha$ increasing tail vortex gradually compressed until it disappearing. Then, analyze the lift and drag characteristics of rotating cylinder under supercritical conditions, there was a linear relationship between the lift coefficient and peripheral speed ratio $\alpha$, the ratio of lift coefficient to peripheral velocity ratio was approximately 2 ; while the drag coefficient changing rules were more complex, it have been analyzed from two aspects of the Reynolds number and rotating speed by author, rotating cylinder's drag coefficient variation with Reynolds number was consistent with stationary cylinder, and with the rotating speed increasing, the drag coefficient was gradually decreased until reducing to a certain value then keeping constant.

\section{ACKNOWLEDGEMENTS}

The work described in this paper was partly supported by the Shenzhen Science and Technology Plans: Key Laboratory Development Projects (Grant No. ZDSYS2014050816154729).

\section{REFERENCES}

E. Achenbach. 1969. Distribution of local pressure and skin friction around a circular in cross-flow up to $\mathrm{Re}=5 \times 106$. Journal of Fluid Mechanics. Vol. 34 (1968) pp.625-639.

F. Diaz, J. Gavaldà, J. G. Kawall, J. G. Keffer, and F. Giralt. 1985. Asymmetrical wake generated by a spinning cylinder [J]. AIAA Journal, Vol. 23, No.1, pp.49-54.

H.G. Magnus. 1853. On the deviation of projectiles; and on a remarkable phenomenon of rotating bodies. Taylor's Foreign Scientific Memoirs, Vol. 804, pp.10.

L. Prandtl. 1926. Application of the "Magnus Effect" to the wind propulsion of ships. NACA Technical Memorandum, Vol. 367, pp.1-12.

M.B. Glauert. 1957. The flow past a rapidly rotating circular cylinder [J]. Proceedings of the Royal Society of London. Series A, Mathematical and Physical Sciences, Vol. 242, No.12, pp.108-115.

M. Coutanceau and C. Menard. 1985. Influence of rotation on the near-wake development behind an impulsively started circular cylinder [J]. Journal of Fluid Mechanics, Vol. 158, pp.399-446.

M.T. Nair, T. K. Sengupta and U.S. Chauhan. 1998. Flow past rotating cylinders at high Reynolds numbers using higher order upwind scheme [J]. Computers \& Fluids, Vol. 27 (1998) No.1, pp. 47-70.

S.J. Karabelas. 2010. Large Eddy Simulation of HighReynolds Number flow past a rotating cylinder. International Journal of Heat and Fluid Flow. Vol. 31 (2010) pp.518-527.

S. Kang, H. Choi, and S. Lee. 1999. Laminar flow past a rotating circular cylinder [J]. Physics of Fluids, Vol. 11, No.11, pp.3312-3321.

S. Mittal. 2004. Three-dimensional instabilities in flow past a rotating cylinder [J]. Journal of Applied Mechanics, Vol. 71, pp.89-95.

W.M. Swanson. 1961. The Magnus effect: a summary of investigations to date. Journal of Basic Engineering Transactions of ASME; Vol. 83, No.3, pp.470.

Y.T. Chew, M. Cheng and S. C. Luo. 1995. A numerical study of flow past a rotating circular cylinder using a hybrid vortex scheme [J]. Journal of Fluid Mechanics, Vol. 299 pp.3571 . 